\title{
Framework of Problem-Based Research: A Guide for Novice Researchers on the Development of a Research-Worthy Problem
}

\author{
Timothy J. Ellis and Yair Levy \\ Nova Southeastern University \\ Graduate School of Computer and Information Sciences \\ Fort Lauderdale, Florida, USA
}

ellist@nova.edu, levyy@nova.edu

\begin{abstract}
This paper introduces the importance of a well-articulated, research-worthy problem statement as the centerpiece for any viable research. The aim of this work is to help novice researchers understand the value of problem-based research by providing a practical guide on the development of a well articulated and viable statement of a research-worthy problem as the starting point for all research. Additionally, this paper explores the interaction of the problem with the other fundamental elements of scholarly research including the research topic, goals, research questions, methodology, results, and conclusions. Scaffolding for articulating a 'research-worthy problem' is provided in the form of a deconstruction of the expression into definitions of its component terms, followed by a discussion of what is not a research-worthy problem. A roadmap on locating problems that could support scholarly research is provided. The theoretical foundation is placed into practice by examining some problem statements and proposing a template for crafting an effective statement.
\end{abstract}

Keywords: Research methodology, research problem, problem-based research, research questions, theory-based research, doctoral education.

\section{Introduction}

The importance of basing research on a well-articulated problem statement is well accepted across disciplines such as information systems, education, and engineering (Creswell, 2005; Hicks \& Turner, 1999; Sekaran, 2003). Unfortunately, just what constitutes a research-worthy problem is not readily apparent, in particular for novice researchers. Although most scholars would agree that not everything that is problematic could serve as the starting point for meaningful research, it is not easy to identify just what does constitute such a problem. According to Ker-

Material published as part of this publication, either on-line or in print, is copyrighted by the Informing Science Institute. Permission to make digital or paper copy of part or all of these works for personal or classroom use is granted without fee provided that the copies are not made or distributed for profit or commercial advantage AND that copies 1) bear this notice in full and 2) give the full citation on the first page. It is permissible to abstract these works so long as credit is given. To copy in all other cases or to republish or to post on a server or to redistribute to lists requires specific permission and payment of a fee. Contact Publisher@InformingScience.org to request redistribution permission. linger and Lee (2000), the identification of the research problem is "the most difficult and important part of the whole [research] process" (p. 15). To a very great extent, Supreme Court Justice Potter Stewart's opinion regarding obscenity seems to describe the feeling of many scholars regarding researchworthy problems: "I shall not today attempt further to define the kinds of material I understand to be embraced ... 
[b]ut I know it when I see it ..." ("Jacobellis v. Ohio", 1964). Unfortunately, such an approach does not appear to be helpful, especially for novice researchers. Thus, this paper attempts to address the critical issue of identifying and establishing the research-worthiness of a problem.

This paper explores the concept of problem-based research beyond the 'know it when I see it' approach. In the balance of this introduction, the argument for basing research on a well-defined problem will be detailed and the interaction of the problem with the other fundamental elements of scholarly research will be explored. The second section builds a scaffold for defining 'research-worthy problem' by deconstructing the expression. The third section places the theoretical foundation laid in the first two sections into practice by examining some effective problem statements. The fourth section provides a practical guide on locating problems that could support scholarly research. Finally, a summary and recommendations are provided.

\title{
Why Problem-Based Research?
}

\author{
"I keep six honest serving-men \\ (They taught me all I knew); \\ Their names are What and Why and When \\ And How and Where and Who" \\ (Kipling, 1902/1988, p. 3)
}

Kipling's (1902/1988) "honest serving-men" have been used to form the basis for enquiry in a number of disciplines. Although the poem has long been the accepted mantra of journalists, it applies equally well to scientific enquiry (Sharp, 2002). A well-structured, properly-reported study must provide answers to all questions regarding the what, why, when, how, where, and who associated with the research. The problem statement offers the context necessary for addressing the why question (Tracy, 2007).

According to Kerlinger and Lee (2000), in essence a scientist is driven by an inner desire to understand "why something is as it is" (p. 15). Moreover, they note that scientists are restless until they find an explanation to the phenomena. One cannot place value on research without a clear understanding of, first, why that research had been conducted (Creswell, 2005). In the case of a great deal of reported research, at least part of the answer to why lies in the need to publish in order to earn a degree, tenure, or promotion (Sharp, 2002). If such rather practical considerations are the only motivators for the research, it is hard to imagine the research producing results of much interest or value. What makes research of interest is how it will impact future research and other researchers, not the author. This impact is most clearly evidenced by addressing a problem that has some manner of impact on the reader.

Answering the question "Why conduct the research?" with an effective statement of the problem doesn't just add meaningfulness to that research; it also serves as the first step in addressing that problem (Sternberg, in press). "A careful statement of the problem goes a long way toward its solution" (Hicks \& Turner, 1999, p. 3). The nature of what is going wrong - the problem - very much sets the parameters for what can be done. Kerlinger and Lee (2000) suggested that "if one wants to solve a [research] problem, one must generally know what the problem is" (p. 24). If, for example, an institution is faced with a situation in which its customer base is shrinking, the appropriate course of action would be radically different if the problem causing that undesirable situation is one of poor service than if it is one of high prices or one of not knowing just what the customers value.

According to Kerlinger and Lee (2000), "adequate statement of the research problem is one of the most important parts of research" (p. 24). They continue to add that the "difficulty of stating a research problem satisfactorily at a given time should not cause one to lose sight of the ultimate 
desirability and necessity of doing so" (Kerlinger \& Lee, p. 24). According to Jacobs (1997), argument to the problem statement "should present how the research builds on previous theory or contributes to the development of new theory, and should describe the likely uses of the knowledge to be gained and the potential importance of these uses" (p. 1). A clear, precise, and well structured problem statement leads to a quality research (Jacobs). O'Connor (2000) noted that a viable research problem is the central and most highly important part of any quality research. Leedy and Ormrod (2005) suggested that the research problem "is the axis around which the whole research effort revolves" (p. 49). The "heart of every research project is the problem. It is paramount to the success of the research effort" (Leedy \& Ormrod, p. 49). Furthermore, "to see the problem with unwavering clarity and to state it in precise and unmistakable terms is the first requirement in the research process" (Leedy \& Ormrod, p. 49). Thus, it appears that there is a clear consensus in literature that identification of a problem is a cornerstone for any quality research. Basing research on a well-articulated, well-supported, and well-argued problem establishes the potential for producing meaningful results. Although studies certainly can and have been conducted without the anchor of a clearly defined problem, such research is essentially flawed; "No amount of good research can find solutions ... if the critical issue or the problem to be studied is not clearly pinpointed" (Sekaran, 2003, p. 69). Therefore, it is warranted that novice researchers should learn to identify a research problem and learn how to properly construct and develop a logical argumentation for a problem statement.

\section{The Role of the Problem in the Structure of the Research}

A research endeavor might best be viewed as a structure that incorporates a number of distinct but related elements including the research problem that drives the study, the goals, research questions, review of the literature, methodology, results, and conclusions. The research problem serves as the starting point for the research and is a unifying thread that runs throughout all the elements of the research endeavor (Leedy \& Ormrod, 2005). Kerlinger and Lee (2000) noted that "without some sort of statement of problem, the scientist can rarely go further and expect the work to be fruitful" (p. 15). A viable research problem is usually noted at the introduction of the research manuscript to identify why the study is important (Creswell, 2005).

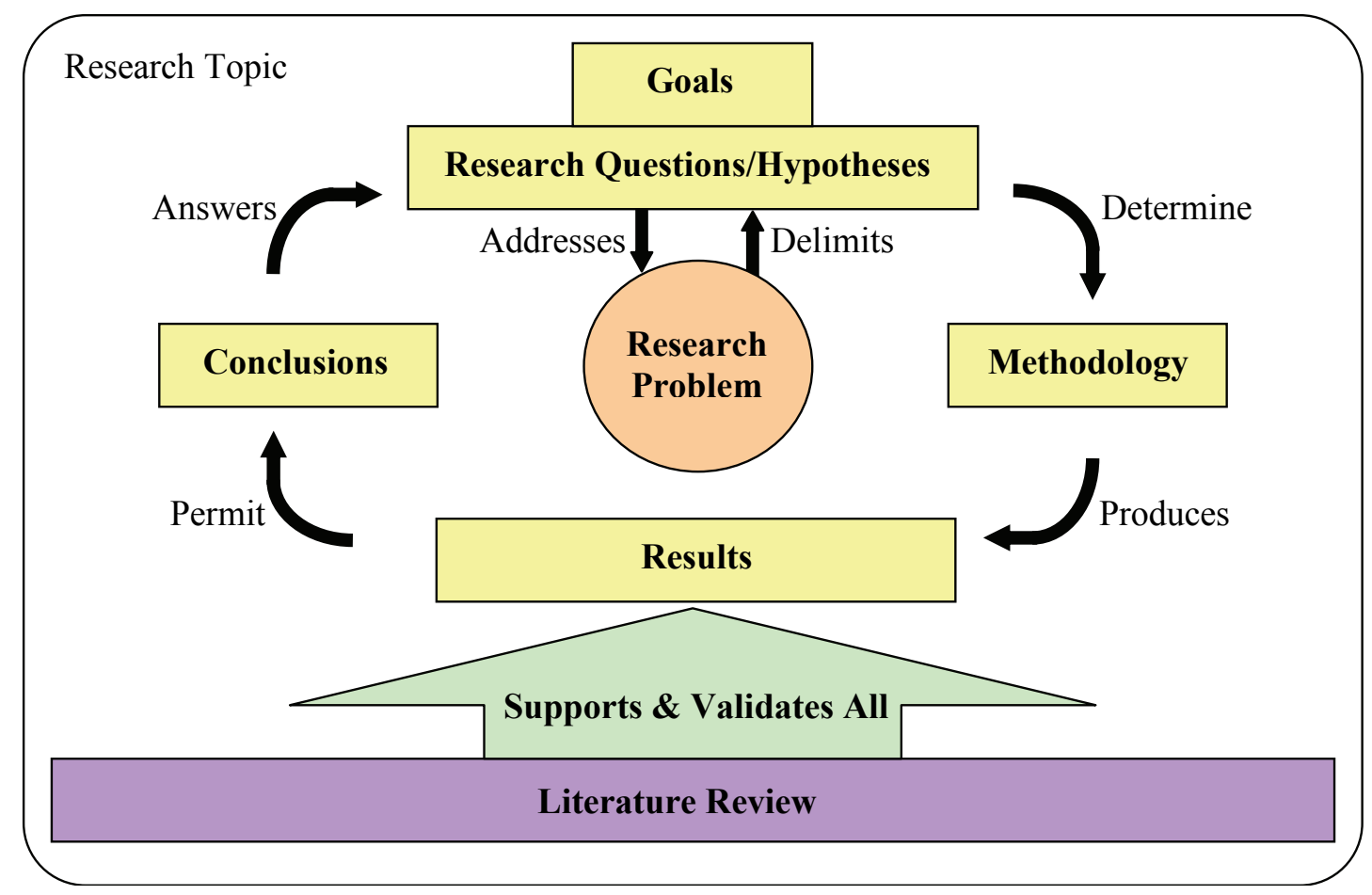

Figure 1: Conceptual Map of the Problem-Based Research Cycle 
The research problem also serves as the basis for the interrelatedness of the distinct elements entailed in research. Figure 1 illustrates this interrelatedness by providing a conceptual map of the problem-based research cycle indicating the centrality of the research problem for the research endeavor. The next few sections examine the relationships between the problem driving the research and the other aspects of the endeavor.

\section{The topic, research problem, goals, and research questions}

As illustrated in Figure 1, the topic is the general domain in which the research is focused. Examples of topics in information systems include business process management, customer relationship management, enterprise information, electronic commerce, e-government, database management, knowledge management, crisis management, information security, human computer interaction, procurement and supply chain management, telecommunication, virtual teams, computer ethics, decision support, online learning, etc. Problems are drawn from the general domain described by the topic.

As shown in Figure 1, there is a two-way relationship between the research problem, the goals, and the associated research questions and/or hypotheses. A research study goal "is the major intent or objective of the study used to address the problem" (Creswell, 2005, p. 62). Research goals essentially detail what the research study intends to do in order to address the problem, thereby answering the question: "What will this study do?" The research goals are operationalized by one or more research questions. Research questions "narrow the purpose [or goal] into specific questions that the researcher would like answered or addressed in the study" (Creswell, 2005, p. 62). By attaining answers to those research questions, the study goals are met and a contribution towards solving the problem is made (Leedy \& Ormrod, 2005). Since problems that can inspire scholarly research are generally rather difficult in their very nature, the contribution towards resolution is generally quite small. However, in order for the research to be at all meaningful, there has to be an identifiable connection between the answers to the research questions and the research problem inspiring the study (Kerlinger \& Lee, 2000). Figure 2 illustrates the relationship among the general topic, research problem, goal, and research questions with an example in the context of knowledge management.

The nature of the research problem being addressed delimits the possible goals for a study. Included in the goal is the type of study being conducted - experimental, developmental, descriptive, etc. Certain characteristics of the problem such as the domain from which it is drawn, how long it has been acknowledged, as well as how much and what type of research has already been conducted on it, impact what type or types of methodology is appropriate (Nunamaker, Chen, \& Purdin, 1991). The type of methodology being used must be appropriate for the nature of the problem driving that research. The methodology produces the results of the study, which in turn produces the evidences needed to permit the conclusions suggested. 


\section{Conceptual \\ Basis \\ $\underline{\text { Artifact }} \quad \underline{\text { Example }}$}

General

$\downarrow$
Topic $\longrightarrow$ Knowledge Management

Research $\longrightarrow$ Difficulty in retaining

Problem organizational knowledge

Goal $\longrightarrow$ A descriptive study to determine the constructs that lead employees to resist the implementation of knowledge management systems (KMSs).

Research $\longrightarrow$ Does employee involvement in Question the development of KMS affect their resistance of KMS implementation?

Figure 2: Relationship Among the Topic, Research Problem, Goals, and Research Questions (Adapted from Creswell, 2005, p. 62)

\section{Bringing in the methodology, results, and conclusions}

Although not directly related to the research problem, the methodology, results, and conclusions of a study are directly impacted by the problem driving the research. As illustrated in Figures 1 and 2, the methodology is structured by the research questions. The methodology is, essentially, the steps that will be taken in order to derive reliable and valid answers to those questions. Professionals from different domains uses their own set of tools to conduct their work activities and develop their desired outcomes (Leedy \& Ormrod, 2005). Researchers are knowledge workers, so they use knowledge tools to conduct their work (Davis \& Parker, 1997). However, the methodology determines the appropriateness of a given research tool. According to Leedy and Ormrod, novice researchers commonly confuse the research methodology with the research tools. A research methodology is defined as "the general approach the researcher takes in carrying out the research project" (Leedy \& Ormrod, p. 14). Research tools, on the other hand, is defined as "a specific mechanism or strategy the researcher uses to collect, manipulate, or interpret data" (Leedy \& Ormrod, p. 14). Research methods commonly used in information systems studies include: descriptive (case study), empirical/quantitative, qualitative, mixed methods (including both qualitative and quantitative), experimental, quasi-experimental, and simulations. Each type of research method encompasses many specific research tools that can be employed to address different research questions (Leedy \& Ormrod, 2005). In general, the methodology must be detailed and address the how, when, where, and who questions. The methodology outlines the types of research tools that the researcher will use to produce the study's results. The study results include the data, or the evidences, that can be used to answer the research questions. The results, in turn, permit the researcher to draw conclusions that are, in fact, the answers to the research questions. Those answers, as mentioned in the previous section, constitute the manner in which the research addresses the underlying problem. 


\section{Building upon a literature foundation}

Although the research problem serves as the starting point for research, the literature review, as illustrated in Figure 1, serves as the foundation upon which that research is built (Levy \& Ellis, 2006). The presence of the research problem is almost always established through the literature review. However, there are occasional visionaries who see research problems long before others, the vast majority of research is built upon problems that are well documented (Leedy \& Ormrod, 2005 , p. 14). The appropriateness of the research goals for the study - the type of research to be conducted and the research questions to be answered - is similarly established through the literature. Likewise, although the methodology is structured by the research questions, its appropriateness must be established through the literature. The manner in which the results are analyzed to produce the study's conclusions is also anchored in the literature.

\section{“Research-Worthy Problem" Deconstructed}

Just as there is an almost universal sentiment that one should lead a "good life", most would agree that one should use research to address problems. Unfortunately, just as there are widely diverging views on just what constitutes a "good life", there is no universal agreement on just what constitutes a "research-worthy problem". Although there is certainly room for honest differences in opinion regarding the research-worthiness of any given problem, it is important that there be a clear understanding of "research-worthy problem" as a construct.

\section{Problem?}

On the surface, "problem" appears to be very easily defined. Dictionary definitions include "a situation, person or thing that needs attention and needs to be dealt with or solved" (Cambridge online dictionary, 2007) and "a question raised for inquiry, consideration, or solution" (Merrianwebster online dictionary, 2007). From a scientific perspective, a research problem is defined as a general issue, concern, or controversy addressed in research. Moreover, a research problem "must integrate concepts and theoretical perspective of the literature into the problem to be addressed" (O'Connor, 2000, p. i). A research problem exists if at least two elements are present. First, the current state differs from the ideal state (Sekaran, 2003). Second, there is not an "acceptable" solution available. The absence of an acceptable solution can entail either there being no solution documented in the literature, or the solutions noted in the literature leading to mixed results or contradictions (i.e. not properly addressing the problem) (Creswell, 2005). Implicit in the definition of a research problem are:

- Problems are active. A missed opportunity does not necessarily constitute a problem. Parents have, for example, a missed opportunity for training toddlers to use automatic weapons, but it is doubtable if anyone in his right mind would seriously consider that as a viable research problem.

- Problems have an impact. A favorite philosophical pursuit of college freshmen is to ponder the question "If a tree falls in a forest and nobody is there to hear it fall, does it make a sound?" One might similarly ask: "How can something be considered a problem if one cannot identify something that is going wrong or, at the very least, not as well as it should?" There are many ways the "going wrong" might be manifested, ranging from physical pain through inconvenience to intellectual angst associated with vacuums or inconsistencies in our understanding. The impact need not be gigantic, but it must be identifiable. In this context, it is important to not confuse topics - areas of interest - with problems. Topics, such as "Implementing a knowledge management system" and "Personalizing training through the use of computer managed instruction", although of interest, lack any impact and cannot serve as the starting point for research. 
- Problems do not have adequate solutions available. It is important to distinguish between problems and other unfavorable situations. One might, for example, have an infestation of ants in his house. Obviously, for most that state of affairs would not correspond with the ideal state, but a quick look through the "exterminators" section of the phone book or Internet would produce a large number of ready solutions to address this unfavorable situation.

\section{Research?}

Research is defined as "the systematic process of collecting and analyzing information (data) in order to increase our understanding of the phenomenon about which we are concerned or interested" (Leedy \& Ormrod, 2005, p. 4). According to Creswell (2005), research is "a process of steps used to collect and analyze information in order to increase our understanding of a topic or issue" (p. 3). The key issue emerging from these definitions is that research must collect and analyze new information and/or data that will enhance the body of knowledge. There are a number of ways in which original research contributions can be made to the body of knowledge, including:

- Establishing causal relationships by conducting a causal-comparative study to address a documented problem.

- Evaluating the efficacy of an approach to addressing a documented problem by conducting an experimental or quasi-experimental study.

- Examining the impact of the element of time on the nature of the documented problem in a longitudinal study.

- Exploring in depth the positive and negative aspects of an approach to address the documented problem in a descriptive study.

- Establishing a method for creating a product that could at least potentially reduce the impact of the documented problem through a developmental study.

- Developing constructs from a pool of observations regarding the causes or characteristics of a well-documented problem through a factor analysis.

- Developing a predictive model in an approach to address a documented problem.

The list of possible approaches to making the necessary original contribution inherent in research could, of course, be extended considerably from the six items listed above. The essential issue, however, remains the same: in order for the endeavor to be considered research, it must clearly present the potential for creating identifiable new knowledge.

Although it might seem self-evident, a brief discussion of "new knowledge" is warranted. In the context of research, "new" refers to information not already present in the body of knowledge in the applicable domain. Just because information might be new to a specific entity - an individual or organization - is irrelevant if it was already present in the research literature of that domain (Hart, 1998). For example, an information systems department might not know how to address the resistance of employees to an enterprise resource planning (ERP) system even though there are numerous studies available about such a problem. A few quick searches in scholarly databases with backward and forward searchers may yield several studies that investigated the very problem and suggested numerous solutions (for additional information on effective literature review including searches techniques such as backward and forward, see Levy \& Ellis, 2006).

\section{Research-Worthiness?}

Unlike the previous terms, "research-worthy" is not definable through a rather simple reference in a dictionary or a scientific definition from peer-reviewed literature. Its meaning can be extrapolated from the concept of "research". As discussed in the previous section, research entails mak- 
ing an original contribution to the applicable body of knowledge. In order for that type of contribution to be possible, certain preconditions are necessary:

- An exhaustive understanding of the body of knowledge related to the field or topic of study. Knowledge of what is known is a prerequisite for identifying that which is unknown (Davis \& Parker, 1997).

- A solid conceptual foundation for the research (Hart, 1998). Although many discoveries are certainly serendipitous, research is intentional, built upon a theoretical basis (Malinski, 2004; Verran, 1997). Research-worthiness entails that there is a real, identifiable conceptual connection between the research problem driving the study and the research being conducted to address that problem (Creswell, 2005). Without that conceptual connection, one would be left with the impression that any new insights resulting from the study were more the result of random luck than scholarly work.

- A "yes" answer to one of the following questions (Creswell, 2005):

○ Will a known gap in the body of knowledge be filled?

- Will previous research be replicated and expanded by looking at a different category of participants, environment, and/or constructs/variables?

- Will previous research be expanded by more thoroughly examining some identifiable aspect?

- Are there specific, identifiable, and documented problems with the currently available solutions?

\section{Research-Worthy Problems Are Not ...}

In the quest for a research-worthy problem, novice researchers should first understand what are not research-worthy problems (Leedy \& Ormrod, 2005). First, research-worthy problems should not be based solely on personal observations and/or experiences. Although a researcher may have a "hunch for the problem", an identifiable literature that documents the problem or literature that documents conflicting results should be the basis for a research-worthy problem (Kerlinger \& Lee, 2000). Second, research-worthy problems should not be based on a comparison of two sets of data for the sake of comparison. Comparing groups is not a research-worthy problem; rather it is a methodology to address a problem. For example, comparing employee productivity before and after ERP training is a valid study, but it does not represent a viable research-worthy problem. A viable research-worthy problem behind such a comparison may be the issue of low productivity level after ERP implementation and the goal of such a study may be to investigate the effectiveness of ERP training on increasing productivity. Hence, the comparison itself doesn't constitute the research-worthy problem but is rather the methodology used to address a problem. Identifying the research-worthy problem behind such a comparison is warranted. Third, researchworthy problems should not be based on a correlation of two sets of data. Leedy and Ormrod noted that a "correlation coefficient is nothing more than a statistic... It tells us nothing about why that relationship exists" (p. 50). An example for a correlation that is meaningless: the growth in egovernment use in North America was found to be highly correlated to the growth in birth rate of elephants in Africa. Such an example provides very little contribution to the body of knowledge because it does not appear to address any viable research-worthy problem. Lastly, researchworthy problems should not be based on an investigation that yields a "yes" or "no" answer. Answers to such questions, again provide very little contribution to the body of knowledge. 


\section{Finding Research-Worthy Problems: Tips From the Field}

\section{From Where Do Research-Worthy Problems Emerge?}

A viable research-worthy problem can emerge from various areas. Leedy and Ormrod observed "problems for research are everywhere. Take a good look at the world around you. Where does your interest lie?" (p. 49). Experienced researchers might identify research-worthy problems by speaking with colleagues, attending scholarly conferences, and by observing a phenomenon (Kerlinger \& Lee, 2000). In many cases, however, seasoned scholars might work on the same problem-area throughout their whole scholarly tenure (Kerlinger \& Lee, 2000; Leedy \& Ormrod, 2005). In general "the problem usually begins with vague and/or unscientific thoughts or unsystematic hunches. It then goes through a series of refinement steps" (Kerlinger \& Lee, 2000, p. 15). Novice researchers may have some "hunches" but it is vital that they properly anchor them in the context of the existing body of knowledge.

Identifying a research-worthy problem for novice researchers should be based on a four-step process. The first key step deals with the process of looking or attempting to identify a potential research-worthy problem. The second key step in the process to identify a potential researchworthy problem is reading the literature and identifying valid scholarly sources. The third key step deals with the process of synthesizing the literature and internalizing the body of knowledge. The fourth step is to consult with others, seeking feedback on the potential research-worthy problem from experts and/or experienced researchers. The following four sections elaborate on this four-step process.

\section{Look}

Personal interests, hunches, and 'gut feelings' are, for most novice researchers, the most reasonable starting point for locating research-worthy problems (Kerlinger \& Lee, 2000). Although, as mentioned above, it is vital to not confuse topics such as knowledge management or computer managed instruction with research-worthy problems, researchers often limit the domain in which they search for a research-worthy problem. Limiting the domain on the basis of personal interest appears to be appropriate and may offer a viable starting point towards identifying a researchworthy problem.

Personal experiences also provide potentially fruitful insight into possible research-worthy problems. Throughout the course of daily activities, individuals encounter situations in which the current state is far from the ideal state; each of these unfortunate situations offers potential as a research problem (Kerlinger \& Lee, 2000). However, it is vital to remember that just because a given organization has a specific problem, doesn't directly constitute that problem as a researchworthy one. It is entirely possible that the organization is simply not aware of a number of solutions to the problem that are well documented in the scholarly literature (Kerlinger \& Lee, 2000).

\section{Read}

Leedy and Ormrod (2005) emphasized that "one essential strategy is to find out what things are already known about your topic of interest; little can be gained by reinventing the wheel" (p. 51). Reading scholarly literature is one of the most important steps in this process. The first critical way that a researcher can identify if a problem observed is indeed a specific instance of a more general research-worthy problem, and not just an example of an organization not being cognizant of well-established solutions, is to read the scholarly literature. The process of reading the scholarly literature must be purposive (Levy \& Ellis, 2006). Such process should include:

- Identifying the leading journals, conference proceedings, and scholars in the domain of interest. 
- Performing exhaustive searches, using techniques such as searching on keywords as well as forward and backward searches on names of scholars cited in articles of interest (Levy \& Ellis, 2006). Additionally, reviewing recently finished doctoral dissertations can provide a 'gold mine' for valid leads to scholarly literature on the topic of interest.

- Identifying "holes" in the body of knowledge identified in the scholarly articles. Moreover, "in addition to telling you what is already known, the existing literature is likely to tell you what is not known in the area - in other words, what still needs to be done" (Leedy \& Ormrod, 2005, p. 51). Most quality research articles will include recommendations for future research in the concluding remarks. Such discussions provide scholarly evidence for what is still needed and what research-worthy problems still exist.

\section{Synthesize}

The viability of a problem as a starting point for scholarly research cannot be established through a single source (Creswell, 2005). It is highly unlikely that any two journal articles would say precisely the same thing about where further research is needed. The researcher must, therefore, develop a research-worthy problem by weaving together the threads derived from a number of sources. The synthesizing process results in creating a whole that in a meaningful way exceeds the sum of its parts (Bloom, Engelhart, Furst, Hill, \& Krathwohl, 1956). Such synthesis usually entails:

- Combining the insights from a number of scholarly articles.

- Integrating the work from different but related fields.

- Composing a generalization based upon multiple specific instances.

\section{Consult}

Once a potential research-worthy problem has been identified, novice researchers are encouraged to seek feedback (Leedy \& Ormrod, 2005). Feedback on the potential research-worthiness of a problem can be done via two main approaches. The first is by seeking feedback from available experienced researchers. According to Leedy and Ormrod, a novice researcher may start to obtain feedback on their proposed research problem simply by asking their own professors: "what needs to be done? What burning questions are still out there? What previous research findings seemingly don't make sense?" (p. 51). The second way to seek feedback from experienced researchers is by attending scholarly and scientific conferences; "by scanning the conference program and attending sessions of interest, they [i.e. novice researchers] can learn "what is hot and what's not" in their field" (p. 51). Following such feedback, researchers may either find that their proposed problem truly presents potential as a point of departure for scholarly research or that they need to go back to the starting point and look again. Figure 3 represents flow-chart of the process of finding research-worthy problems. 


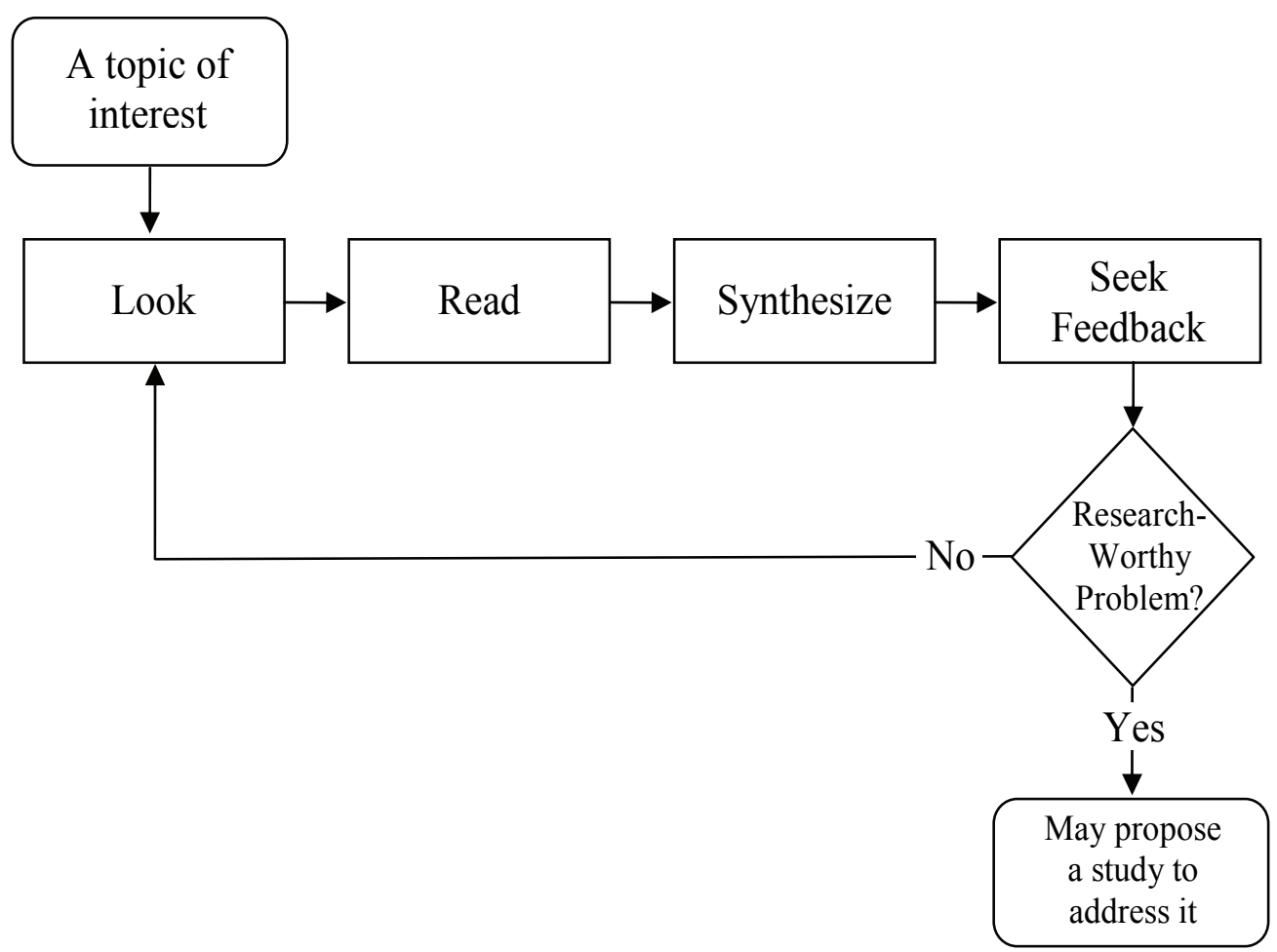

Figure 3: Process of Finding a Research-Worthy Problem

\section{A Template for Crafting a Problem Statement}

Prior to discussion about the problem statement, novice researchers should fully understand the difference between the statement of the problem and the problem statement. A statement of the problem is one or two sentences that outline the problem that the study addresses. The statement of the problem should briefly address the question: What is the problem that the research will address? (See number 1 in Figure 4). However, the problem statement is the statement of the problem and the argumentation for its viability (see more about argumentation theory and development of valid argument in Levy and Ellis, 2006). The problem statement should address all six questions: what, how, where, when, why, and who (see Figure 4). Creswell (2005) observed that the problem statement should be stated in the introductory sections of the research manuscript and provide the rationale for its importance by "developing justifications for studying it [the research problem]" (p. 8). Another clarification should be made about the confusion between the statement of the problem and the main research question guiding the study. Research questions are formulated in a question format and are narrowed enough to identify the variables or construct under study, while the statement of the problem should not be noted in a question format and should be in a higher theoretical level derived from the overall problem the study addresses (Figure 2). 
1. What: In no more than two sentences, what is the problem that the research will address? Remember, a problem is, essentially, something that is 'going wrong'.

Who: List three current, peer-reviewed references that support the presence of that problem and briefly describe the nature of that support.

2. How, Where, and When: Again, in no more than two sentences, describe the impact of the problem. How are people or researchers' understanding negatively impacted by the problem? When and where is the problem evident?

Who: List three current, peer-reviewed references that support the impact of the problem that the research proposes addressing and briefly describe the nature of that support.

3. Why: In no more than two sentences, identify the conceptual basis for the problem. That is, what does the literature outline as the cause of the problem?

Who: List three current, peer-reviewed references that support the conceptual basis of the problem and briefly describe the nature of that support.

Figure 4: Problem Statement Template

The development of a problem statement "is an exercise in logic" (O'Connor, 2000, p. i). Identifying a research-worthy problem is far from a mechanical process and depends on a combination of inspiration, perspiration, and logical argumentation. There are, as described above, steps that can be taken to facilitate the process. The difficulty of articulating a problem statement that can indeed serve as a starting point for scholarly research transcends simple solution. However, "a well developed problem statement appears simple. But writing a good problem statement is far from effortless. Rather it is a complex activity related to preparing a logical argument" (O'Connor, 2000, p. i). Leedy and Ormrod (2005) suggested that the starting point of the problem statement is in a well-articulated statement of the problem that "must first be expressed with the utmost precision" (p. 49).

A procedure in developing a problem statement cannot be a complete solution to this difficulty. There are some meaningful landmarks that can provide insight into progress towards developing such a statement. The Problem Statement Template (Figure 4) provides an overview of how the six questions of what, how, where, when, why, and who, should be addressed. When identifying a research-worthy problem based on the process noted above, researchers may find it easier to address these six questions.

\section{Exploring a Sample Problem Statement}

A well-articulated problem statement should answer the questions outlined in the Problem Statement Template (Figure 4) in several well-focused sentences. Since research is meaningful only in the context of the problem being addressed, a succinct yet complete statement of the problem should be one of, if the not first thing the reader encounters (Creswell, 2005). The exact wording of a problem statement is, of course, dependent on the research domain and the nature of the problem being addressed. However, Leedy and Ormrod (2005) noted that the key to successful documentation of a viable research problem is articulating precisely what the proposed problem is. They noted that researchers should say exactly what they mean and that researchers "should not assume that others will be able to read your mind" (p. 53). Moreover, they noted that failure 
to carefully select the appropriate words "can have grave results for your status as a scholar and a researcher" (Leedy \& Ormrod, p. 53). Accordingly, this section will explore the specifics of what constitutes a viable problem statement by first presenting as a "straw man" a rather typical example of a faulty problem statement, followed by an analysis of the faults in the "straw man", and concluding with a revised statement of the problem.

\title{
A Sample of a Faulty Problem Statement
}

Figure 5 presents a rather typical faulty problem statement submitted by a novice researcher as part of an initial research proposal. Please note that, since the material in Figure 5 is presented only as an example, the citations listed in the example are not included in the references of this paper.

\begin{abstract}
"One main obstacle of knowledge management is the lack of developed culture in an organization to ensure the acceptance of a knowledge management system (BecerraFernandez \& Sabherwal, 2001; Bossen \& Palsgaard, 2005; Kaweevisultrali \& Chan, 2007; Pumareja \& Sikkel, 2005). Non-acceptance of knowledge management system occurs with limited or no support when the proper culture for utilizing knowledge management is not practiced in the organization (Gottschalk, 2000; Kruizinga, van Heijst, \& van der Spek, 1996; Swan, Newell, \& Robertson, 2000). The basis for the problem is the lack of aligning knowledge management systems with the business strategy in order to develop knowledge management culture in the organization (Braganza \& Mollenkramer, 2002; Chua \& Lam, 2005; Storey \& Barnett, 2000).”
\end{abstract}

Figure 5: Example of Faulty Problem Statement

\section{The Problem Statement Analysis}

As is often the case, the problem statement noted in Figure 5 touches upon a research area with potential, but does not really present the focus and depth necessary to serve as a starting point for problem-based research. Fitting the information presented into the Problem Statement Template (Figure 4) offers some insight on elevating this initial idea into a viable problem statement that properly articulates the research-worthy problem. The following discussion is based on the example of faulty problem statement noted in Figure 5.

1. What: In no more than two sentences, what is the problem that the research will address? Remember, a problem is, essentially, something that is 'going wrong'.

The statement "One main obstacle of knowledge management is the lack of developed culture in an organization to ensure the acceptance of a knowledge management system" - does not really establish the presence of a problem. It does describe a situation, but there is no indication that that situation is indicative of "something going wrong". What difference does it make if knowledge management were blocked in an organization or not?

Who: List three current, peer-reviewed references that support the presence of that problem and briefly describe the nature of that support.

Although there are citations offered in support of the statement describing the impact of culture on success of knowledge management initiatives (addressing the question of 'says 
who?'), there is no indication of what was contained in the articles cited and how they supported the problem. It is not enough to just point to the literature; there must be enough discussion to establish just how the literature supports the point being made. When using references to back a claim, novice researchers should also ensure they address the 'based on what?' question. In other words, if references are used, there must be a clear argument in the problem statement to the evidences each reference provides (for additional discussion about argumentation theory see Levy and Ellis, 2006).

2. How, Where, and When: Again, in no more than two sentences, describe the impact of the problem. How are people or researchers' understanding negatively impacted by the problem? When and where is the problem evident?

There is no indication in the example provided in Figure 5 of why a failed knowledge management implementation is of any concern. Even though the impact of such a failure might seem intuitively obvious to the novice researcher who wrote the problem statement, it still must be articulated for the benefit of the reader and the proper development of the argument for the research-worthy problem.

3. Why: In no more than two sentences, identify the conceptual basis for the problem. That is, what does the literature might be the cause of the problem?

The statement "The basis for the problem is the lack of aligning knowledge management systems with the business strategy in order to develop knowledge management culture in the organization" offers a conceptual explanation for a problem, but it is not clear just what problem. The text should explicitly identify the conceptual basis for the problem.

Who: List three current, peer-reviewed references that support the conceptual basis of the problem and briefly describe the nature of that support.

Similarly to the issue noted in the lack of addressing the "who" in number one above, although there are citations offered in support of the statement that attempted to describe the conceptual basis for the problem (addressing the question of "says who?"), there is no indication of what was contained in the articles cited and how they identify the conceptual basis for the problem. Novice researchers should ensure the references provided address the "based on what?"

\section{The Revised Problem Statement}

Figure 6 presents a rephrased problem statement based on the one noted in Figure 5 and attempts to better describe the research being proposed by more effectively addressing the questions included in the Problem Statement Template. As noted before, since the material in Figure 6 is presented only as an example, the citations listed in the example are not included in the references of this paper. 
"Knowledge management systems (KMS) have proven to be quite difficult to implement (Becerra-Fernandez \& Sabherwal, 2001; Bossen \& Palsgaard, 2005; Kaweevisultrali \& Chan, 2007; Pumareja \& Sikkel, 2005). According to Pumareja and Sikkel, such difficulty was observed even when careful attention is paid to involving upper management and key stakeholders in the design and implementation process. Additionally, some difficulties in KMS implementation have been observed due to technological barriers (Bossen \& Palsgaard) and users' perceived knowledge satisfaction (Becerra-Fernandez \& Sabherwal). According to Kaweevisultrali and Chan, cultural values place greater emphasis on cooperation and team effort than individual goal attainment during KMS implementation. The benefits of successful KMS implementations have been documented. Wong, Crowder, Wills, and Shadbolt (2006) found that KMS implementation reduces product development time, while Beis, Loucopoulos, Pyrgiotis, and Zografos (2006) found that such implementation creates complex models to facilitate organizational change. However, KMS implementation coupled with the costs associated with failed attempts like lost revenues and reduced employee confidence make effective implementation of KM efforts vital (Braganza \& Mollenkramer, 2002). Although a number of factors have been suggested as important elements in impacting the success of a KMS, the impact of organizational culture appears as a common thread (Bossen \& Palsgaard; Kaweevisultrali \& Chan; Pumareja \& Sikkel). Unfortunately, very little attention has been given in literature to exactly what constitutes the optimal organizational culture for an effective KMS and how to foster that culture."

Figure 6: Example of a Viable Problem Statement

\section{Summary and Recommendations}

This paper addressed a vital issue for novice researchers: the development of a statement establishing the presence of a valid, research-worthy problem. The paper started with an argument for the value of problem-directed research and the centrality of a well-articulated problem statement in research. A discussion of the relationship between the research problem and other fundamental elements of scholarly research such as the research topic, goals, research questions, methodology, results, and conclusions followed. The phrase 'research-worthy problem' was deconstructed and definitions provided for each component, amplified by a discussion of what does not constitute research-worthiness. The paper concluded by outlining the potential sources for locating researchworthy problems and a practical guide for developing an effective problem statement. A template for crafting a problem statement is provided with an example of a faulty problem statement followed by the same example revised to properly indicate a viable problem.

Although it appears easy to establish the value of problem-directed research, it is much more difficult to prescribe just how the novice researcher can in fact develop an acceptable problem statement. Problem identification entails an element of creativity and, therefore, defies a mechanical, highly structured process orientation. The process described in this paper might best be viewed as a roadmap that provides a framework in which a problem statement can be developed rather than a Global Positioning System (GPS) that provides detailed, specific directions, on finding one. Just as, when using a roadmap one can still get lost, it is important to remember that following the framework described above will not guarantee development of a viable problem statement that can serve as the starting point for scholarly research. Although looking at personal experiences, reading and synthesizing the scholarly literature, as well as consulting with domain experts and experienced researchers have proven to be successful in identifying and refining a research-worthy problem, such steps are neither a magic formula for producing a problem statement nor the exclusive method for doing so. Further exploration from experienced researchers in 
just how they identify problems that can serve as points of departure for scholarly research would be of interest. Similarly, the Problem Statement Template illustrated in Figure 4 is certainly not prescriptive and cannot be used as a 'fill in the blanks' form. There would be little argument that a problem statement supported by the literature is a necessary starting point for scholarly research. The process of specifying that problem statement can certainly vary widely from that illustrated in Figure 6. Further exploration from experienced researchers in just how they explicate a problem statement would be of interest.

\section{References}

Bloom, B. S., Engelhart, M. D., Furst, E. J., Hill, W. H., \& Krathwohl, D. R. (Eds.). (1956). Taxonomy of educational objectives, the classification of educational goals, handbook $i$ : Cognitive domain. New York, NY: Longmans.

Cambridge online dictionary. (2007). Retrieved August 10, 2007, from http://dictionary.cambridge.org/define.asp?key=63066\&dict=CALD

Creswell, J. W. (2005). Educational research: Planning, conducting, and evaluating quantitative and qualitative research (2nd ed.). Upper Saddle River, NJ: Pearson.

Davis, G. B., \& Parker, C. A. (1997). Writing the doctoral dissertation: A systematic approach (2nd ed.). Hauppauge, NY: Barrons Educational Series.

Hart, C. (1998). Doing a literature review: Releasing the social science research imagination. London, UK: Sage Publications.

Hicks, C. R., \& Turner, K. V. (1999). Fundamental concepts in the design of experiments. New York, NY: Oxford University Press.

Jacobellis v. Ohio, 378 U.S. 184, 197 (1964).

Jacobs, R. L. (1997). HRD is not the research problem. Human Resource Development Quarterly, 8(1), 1-4.

Kerlinger, F. N., \& Lee, H. B. (2000). Foundations of behavioral research (4th ed.). Holt, NY: Harcourt College Publishers.

Kipling, R. (1988). The elephant's child. Hong Kong: Voyager Books. (Original work published in 1902)

Leedy, P. D., \& Ormrod, J. E. (2005). Practical research: Planning and design (8th ed.). Upper Saddle River, NJ: Prentice Hall.

Levy, Y., \& Ellis, T. J. (2006). A systems approach to conduct an effective literature review in support of information systems research. Informing Science Journal, 9, 181-212. Retrieved from http://inform.nu/Articles/Vol9/V9p181-212Levy99.pdf

Malinski, V. M. (2004). Nursing theory-based research: Parse's theory. Nursing Science Quarterly, 17(3), 201-207.

Merrian-webster online dictionary. (2007). Retrieved August 10, 2007, from http://www.merriamwebster.com/dictionary/problem

Nunamaker, J. F., Chen, M., \& Purdin, T. D. M. (1991). Systems development in information systems research. Journal of Management Information Systems, 7(3), 89-106.

O'Connor, B. N. (2000). Letter from the editor: The research problem. Information Technology, Learning, and Performance Journal, 18(2), i-ii.

Sekaran, U. (2003). Research methods for business (4th ed.). Hoboken, NJ: John Wiley \& Sons.

Sharp, D. (2002). Kipling's guide to writing a scientific paper. Croatian Medical Journal, 43(3), 262-267.

Sternberg, R. J. (in press). The importance of problem-driven research: Bringing Wachtel's argument into the present. Applied and Preventive Psychology. 
Tracy, S. J. (2007). Taking the plunge: A contextual approach to problem-based research. Communication Monographs, 74(1), 106-111.

Verran, J. A. (1997). The value of theory-driven (rather than problem-driven) research. Seminars for Nurse Managers, 5(4), 169-172.

\section{Biographies}

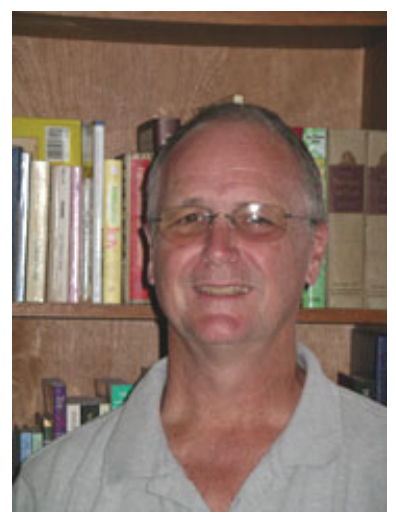

Dr. Timothy Ellis obtained a B.S. degree in History from Bradley University, an M.A. in Rehabilitation Counseling from Southern Illinois University, a C.A.G.S. in Rehabilitation Administration from Northeastern University, and a Ph.D. in Computing Technology in Education from Nova Southeastern University. He joined NSU as Assistant Professor in 1999 and currently teaches computer technology courses at both the Masters and Ph.D. level in the School of Computer and Information Sciences. Prior to joining NSU, he was on the faculty at Fisher College in the Computer Technology department and, prior to that, was a Systems Engineer for Tandy Business Products. His research interests include: multimedia, distance education, and adult learning. He has published in several technical and educational journals including Catalyst, Journal of Instructional Delivery Systems, and Journal of Instructional Multimedia and Hypermedia. His email address is ellist@,nova.edu. His main website is located at http://www.scis.nova.edu/ ellist/

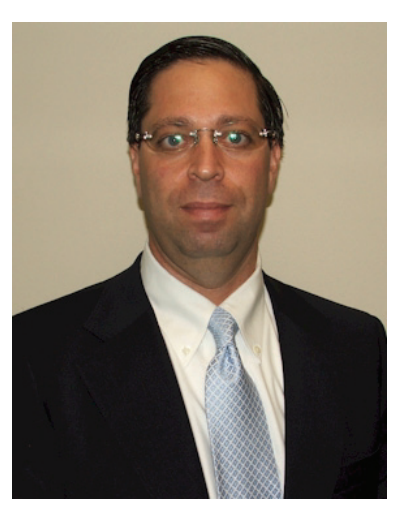

Dr. Yair Levy is an associate professor at the Graduate School of Computer and Information Sciences at Nova Southeastern University. During the mid to late 1990s, he assisted NASA to develop e-learning systems. He earned his Bachelor's degree in Aerospace Engineering from the Technion (Israel Institute of Technology). He received his MBA with MIS concentration and Ph.D. in Management Information Systems from Florida International University. His current research interests include cognitive value of IS, of online learning systems, effectiveness of IS, and cognitive aspects of IS. Dr. Levy is the author of the book "Assessing the Value of e-Learning systems". His research publications appear in the IS journals, conference proceedings, invited book chapters, and encyclopedias. Additionally, he chaired and cochaired multiple sessions/tracks in recognized conferences. Currently, Dr. Levy is serving as the Editor-in-Chief of the International Journal of Doctoral Studies (IJDS). Additionally, he is serving as an associate editor for the International Journal of Web-based Learning and Teaching Technologies (IJWLTT). Moreover, he is serving as a member of editorial review or advisory board of several refereed journals. Additionally, Dr. Levy has been serving as a referee research reviewer for numerous national and international scientific journals, conference proceedings, as well as MIS and Information Security textbooks. He is also a frequent speaker at national and international meetings on MIS and online learning topics. To find out more about Dr. Levy, please visit his site: http://scis.nova.edu/ levyy/ 